\title{
Características Sintomatológicas de Crianças com Autismo e Regressão da Linguagem Oral
}

\author{
Bárbara Backes ${ }^{1}$ \\ Regina Basso Zanon \\ Cleonice Alves Bosa \\ Universidade Federal do Rio Grande do Sul
}

\begin{abstract}
RESUMO - O presente estudo investigou as características da regressão da linguagem oral e da sintomatologia em crianças pré-escolares com Transtorno do Espectro Autista, relacionando-o com os pressupostos da perspectiva sociopragmática. Foi utilizado um banco de dados com 150 crianças norte-americanas, sendo as informações referentes à regressão da linguagem oral e à sintomatologia obtidas por meio da Autism Diagnostic Interview - Revised. Trinta crianças preencheram os critérios para participação neste estudo. Destas, seis apresentaram regressão das habilidades de linguagem oral, com uma média de idade de 25 meses para o início da perda. Em relação à sintomatologia, alguns comportamentos destacaram-se por apresentarem maior comprometimento, como o direcionamento da atenção, a conversação recíproca e o uso repetitivo de objetos.

Palavras-chave: autismo, sintomas, linguagem, regressão
\end{abstract}

\section{Symptomatic Characteristics of Children with Autism and Language Regression}

\begin{abstract}
This study aimed to identify characteristics of oral language regression and characteristics of Autism Spectrum Disorder's symptomatology in preschool children with the disorder, relating it to the assumptions of the sociopragmatic approach. A database of 150 North American children was used, and the information regarding the regression and symptomatology were obtained from the Autism Diagnostic Interview - Revised. Thirty of these children met the criteria for participation in this study. Among them, six presented regression of oral language skills, with a mean age of onset of 25 months. Regarding the symptomatology, some behaviors presented a more severe impairment, like the ability of directing attention, reciprocal conversation and repetitive use of objects.
\end{abstract}

Keywords: autism, symptoms, language, regression

O Transtorno do Espectro Autista (TEA) é uma condição neurodesenvolvimental, que se apresenta nos primeiros anos de vida da criança (American Psychiatric Association [APA], 2013). A etiologia do TEA ainda é desconhecida, mas pesquisas têm encontrado correlatos neurobiológicos e genéticos (Rutter, 2011). Além disso, dados epidemiológicos demonstram uma prevalência de um em cada 68 nascimentos (Centers for Disease Control and Prevention, 2014), sendo que esta tem aumentado nas últimas décadas, o que pode ser explicado pela expansão dos critérios diagnósticos, pelo incremento dos serviços de saúde relacionados ao transtorno e pela mudança na idade do diagnóstico, entre outros fatores (Fombonne, 2009).

O TEA caracteriza-se pela presença de déficits sociocomunicativos e de padrões de comportamentos repetitivos e restritos (APA, 2013), sendo que o grau de comprometimento dessas áreas varia de forma significativa (Volden, Coolican, Garon, White, \& Bryson, 2009). Conforme os critérios diagnósticos presentes no DSM-V (APA, 2013), as alterações na dimensão sociocomunicativa, por exemplo, são encontradas na reciprocidade socioemocional, em comportamentos comunicativos verbais e não verbais e

1 Endereço para correspondência: Rua Ramiro Barcelos, 2600, Bairro, Santa Cecília, Universidade Federal do Rio Grande do Sul, Intituto de Psicologia, Porto Alegre, RS, Brasil. CEP. 90.035-033. E-mail: barbara. edas@gmail.com no estabelecimento e manutenção de relacionamentos. Já a presença de comportamentos repetitivos e restritos pode se manifestar através de estereotipias e repetições nos movimentos motores, no uso de objetos e na fala, além de interesses restritos, adesão excessiva e rígida a rotinas e hipo ou hipersensibilidade a inputs sensoriais (APA, 2013).

Pesquisas têm demonstrado que déficits na comunicação de crianças com TEA apresentam-se geralmente antes dos dois primeiros anos de vida (Landa, 2007; Rapin \& Dunn, 2003), sendo que a ausência das primeiras palavras e frases é um dos principais motivos de preocupação dos pais de crianças com o transtorno (McConkey, TruesdaleKennedy, \& Cassidy, 2009; Zanon, 2012). Em função disso, a linguagem é um campo investigativo bastante importante no que concerne ao TEA. Estima-se, por exemplo, que entre $25 \%$ e $50 \%$ dos indivíduos com o transtorno não adquiram linguagem funcional ao longo da vida (Klinger, Dawson, \& Renner, 2002). Entretanto, ainda há aspectos referentes ao desenvolvimento linguístico de crianças com TEA que são pouco compreendidos, como é o caso da regressão desenvolvimental. Operacionalmente, esta é definida como a perda rápida e pronunciada de habilidades de interação social e de comunicação, após um período de desenvolvimento aparentemente típico, durante o primeiro ano de vida da criança (Matson \& Kozlowski, 2010; Rogers, 2004). A etiologia da regressão desenvolvimental é desconhecida, embora esta tenha sido associada a causas genéticas (Molloy, 
Keddache, \& Martin, 2005) e à epilepsia (Zhang, Xu, Liu, $\mathrm{Li}, \& \mathrm{Xu}, 2012)$. Especificamente em relação à epilepsia, há um número expressivo de estudos que investigou a relação entre essa condição e a regressão (Baird et al., 2008; Hansen et al., 2008; Jones \& Campbell, 2010). Para Tuchman (2006), apesar de sua aparente importância, a relação entre TEA, regressão e epilepsia ainda não é clara na literatura, permanecendo pouco compreendida e controversa.

Embora não exista um consenso, operacionalmente a regressão de habilidades de linguagem oral tende a ser definida como a perda do uso comunicativo de três a cinco palavras, com exceção de 'papa' e 'mama' (Baird et al., 2008; Lord, Shulman, \& DiLavore, 2004; Meilleur \& Fombonne, 2009). Além disso, em geral, os estudos têm utilizado como critério para definição do estabelecimento da perda, que tanto o período anterior à regressão da linguagem quanto o tempo de duração da mesma seja igual ou superior a três meses (Baird et al., 2008; Meilleur \& Fombonne, 2009).

Há uma tendência de grande parte dos estudos acerca do fenômeno da regressão desenvolvimental utilizar a Autism Diagnostic Interview-Revised (ADI-R) para investigar a perda de habilidades em diferentes áreas do desenvolvimento, como linguagem, interação social e brincadeira (Baird et al., 2008; Jones \& Campbell, 2010; Meilleur \& Fombonne, 2009; Molloy et al. 2005; Pickles et al., 2009). Conforme um estudo meta-analítico, a regressão desenvolvimental afeta cerca de $32 \%$ das crianças com TEA, quando elas têm em média 21 meses de idade (Barger, Campbell, \& McDonough, 2013). Além disso, algumas pesquisas revelam que a regressão desenvolvimental acomete, mais comumente, as habilidades de linguagem oral (Baird et al., 2008; Siperstein \& Volkmar, 2004; Wiggins, Rice, \& Baio, 2009), ocorrendo em cerca de $20 \%$ dos indivíduos com TEA (Lord et al., 2004; Siperstein \& Volkmar, 2004; Wiggins et al., 2009), mas esse percentual varia nos diferentes estudos. Por exemplo, na investigação de Meilleur e Fombonne (2009), apenas 9\% dos participantes com TEA apresentaram perda isolada de habilidades de linguagem. Além disso, algumas pesquisas demonstraram a coocorrência de regressão da linguagem oral com perda de habilidades sociais (Baird et al., 2008; Hansen et al., 2008; Meilleur \& Fombonne, 2009).

A falta de consenso sobre diferentes aspectos da regressão desenvolvimental, como apresentado anteriormente, aponta para a necessidade de se ampliar as investigações sobre a ocorrência desse fenômeno na população com TEA e suas peculiaridades, em diferentes estudos. Além disso, observa-se uma lacuna quanto a explicações teóricas acerca da perda de habilidades de linguagem, já que grande parte dos estudos nessa área tem cunho epidemiológico. Nesse sentido, a perspectiva sociopragmática (Tomasello, 1999/2003) poderia contribuir para a compreensão desse fenômeno por considerar a inter-relação no desenvolvimento das habilidades linguísticas e sociais, ambas frequentemente afetadas pela regressão desenvolvimental (Backes, 2012).

Ademais, alguns estudos têm demonstrado haver associação entre a regressão da linguagem oral e a gravidade da "tríade sintomatológica" do TEA (interação social, comunicação e comportamentos repetitivos e estereotipados), o que pode tornar a regressão um fator de risco para o desenvolvimento de crianças com o transtorno (Meilleur
\& Fombonne, 2009). Destaca-se, também, que a perda de palavras constitui-se em um fenômeno importante para a identificação precoce de traços autísticos, já que parece ser característica, embora não universal, de crianças com TEA (Baird et al., 2008; Lord et al., 2004; Luyster et al., 2005). Assim, considera-se importante conhecer as características sintomatológicas dessas crianças, de forma descritiva e embasada em teorias do desenvolvimento.

\section{Pressupostos da Teoria Sociopragmática}

Conforme Tomasello (1999/2003), grande parte dos comprometimentos sociocomunicativos apresentados por crianças com autismo pode ser explicada em função da dificuldade que essas têm de compreender os outros como agentes intencionais e mentais. Para o autor, esta competência, exclusiva dos seres humanos, é a chave sociocognitiva que permite às crianças participarem da cognição humana, interagindo com o meio social de maneira plena e desenvolvendo formas únicas de representações simbólicas.

Nesse processo, destaca-se a Atenção Compartilhada (AC), que é uma habilidade desenvolvida em situações de interação social triádica em que a criança e o adulto dividem experiências em relação a um terceiro objeto/ evento/símbolo referente. Salienta-se que o direcionamento da atenção do outro, numa cena de iniciativa de $\mathrm{AC}$, exige alto grau de complexidade, estabelecendo-se entre os 13 e 15 meses (Tomasello, 1999/2003). Para o autor, a AC é fundamental ao desenvolvimento da linguagem, já que possibilita que a criança compreenda os outros como agentes intencionais e, consequentemente, entenda a intenção do ato sociocomunicativo. Essa compreensão emerge aos 12 meses e se estabelece em torno dos 18 meses, servindo como base para a imitação com inversão de papéis, principal forma de aprendizagem de símbolos linguísticos. Através desta, a criança aprende a utilizar um símbolo comunicativo dirigido ao adulto da mesma forma como este o fez em relação a ela.

Nessa assertiva, Tomasello (1999/2003) considera o período dos 9 aos 18 meses como um dos mais críticos para o desenvolvimento sociocomunicativo infantil. É justamente nesse entremeio que a criança aprende as características básicas dos símbolos linguísticos, isto é, a intersubjetividade e a perspectiva. Estes são intersubjetivos, pois todos os agentes envolvidos no intercâmbio discursivo compreendem seu propósito comunicativo; e perspectivos porque podem variar de acordo com esse propósito. Ademais, é nesse período que ocorre a aquisição dos primeiros marcos linguísticos. Conforme o autor, aos 12 meses de idade surgem as holófrases, que são expressões linguísticas compostas por apenas uma unidade. Aos 18 meses aparecem as construçõespivô, constituídas por dois vocábulos, e aos 22 meses já se observam formulações linguísticas organizadas em torno de verbos, denominadas por Tomasello (1999/2003) de construções verbais insuladas. Ressalta-se que esse processo aquisitivo ocorre simultaneamente ao desenvolvimento sociocognitivo, influenciando e sendo influenciado por este. Em função disso, o autor destaca a importância do desenvolvimento da linguagem, uma vez que este fornece à 
cognição infantil a flexibilidade exigida durante as interações com parceiros sociais, que, por sua vez, se apresentam comprometidas em crianças com TEA.

Com base no que foi apresentado anteriormente, destaca-se que: (a) a regressão da linguagem oral parece ser característica de crianças com TEA; (b) esse fenômeno pode constituir-se em um fator de risco para o desenvolvimento posterior infantil; (c) não há consenso quanto a diferentes aspectos relacionados à perda de habilidades, como a coocorrência com epilepsia; (d) há uma lacuna de explicações teóricas acerca do fenômeno da regressão desenvolvimental e suas implicações; (e) a perspectiva sociopragmática fornece subsídios importantes para a compreensão do desenvolvimento infantil e suas alterações. Assim, o presente estudo teve como objetivo investigar as características de regressão das habilidades de linguagem oral e da sintomatologia de crianças pré-escolares com TEA nas áreas que definem o transtorno. Mais especificamente, objetivouse explicar essas características a partir dos pressupostos postulados pela perspectiva sociopragmática. Além disso, buscou-se identificar a porcentagem de crianças que apresentou regressão, média de idade de início da regressão, nível de linguagem antes da perda, tipo de habilidades de linguagem perdidas, coocorrência com epilepsia e a possível perda de habilidades em outras áreas do desenvolvimento (motoras, autocuidado, brincadeira e sociabilidade).

\section{Método}

\section{Delineamento e Participantes}

Foi realizada uma pesquisa exploratória, descritiva, de cunho retrospectivo. Este estudo está vinculado a um projeto que investiga os preditores do desenvolvimento social de crianças com TEA, utilizando um banco de dados internacional composto por 150 crianças, em idade préescolar, atendidas no Cincinnati Children's Medical Center (CCHMC), Ohio (Estados Unidos da América), entre 2008 e 2009.

Desta forma, foram incluídas no estudo crianças em idade pré-escolar, caucasianas, com diagnóstico de Transtorno Autista e com os dados completos nas medidas investigadas. Como critério de exclusão estabeleceu-se a presença de deficiências físicas e sensoriais associadas, assim como os demais diagnósticos constituintes do TEA.

\section{Instrumentos}

A ADI-R (Lord, Rutter, \& Le Counter, 1994) é uma entrevista padronizada, semiestruturada, administrada aos pais ou cuidadores de crianças com suspeita de TEA. Possui 93 itens que investigam os comprometimentos sociocomunicativos e a presença de comportamentos repetitivos e estereotipados. As informações fornecidas pelos pais ou cuidadores deve, então, ser codificada em escores de 0 a 2, ou 0 a 3 dependendo do item, sendo que 0 implica em um comportamento menos comprometido e 2 ou 3 a comportamentos mais atípicos. A ADI-R é considerada um instrumento padrão-ouro para o diagnóstico de TEA, sendo que o entrevistador deve passar por um treinamento prévio que o habilitará a administrá-la e a codificar seus resultados.

Nesta pesquisa, a regressão das habilidades de linguagem oral foi avaliada com base nos itens 11 a 19 da ADI-R, uma vez que estes investigam especificamente a perda dessas habilidades, a idade em que esta ocorreu, sua duração e a coocorrência com doenças orgânicas (e.g., epilepsia). Para fins investigativos, a linguagem oral adquirida foi definida como o uso de no mínimo cinco palavras diferentes com significado, além de 'papa' e 'mama', usadas rotineiramente por um período mínimo de três meses. Para se considerar a perda de linguagem oral como regressão desenvolvimental deve haver perda de no mínimo cinco palavras com significado por um período superior a três meses. Já a regressão de outras habilidades foi investigada a partir dos itens 20 a 28, juntamente com a idade de sua ocorrência, sua duração e a coocorrência com doenças orgânicas.

A sintomatologia do TEA, referente às habilidades de interação social e comunicação, bem como à presença de comportamentos repetitivos e estereotipados, foi acessada pelos itens contemplados no algoritmo da ADI-R, por se tratarem de questões com força diagnóstica.

\section{Procedimentos e Considerações Éticas}

A autorização para o uso do banco de dados nesta pesquisa partiu de um projeto financiado pela Capes/ Fulbright, desenvolvido pelo Programa de Pós-Graduação em Psicologia da Universidade do Rio Grande do Sul em conjunto com o Centro de Transtornos do Desenvolvimento do CCHMC. Portanto, a autora do projeto principal, do qual este estudo faz parte, frequentou um workshop acerca dos aspectos éticos envolvidos em pesquisas desta natureza. Além disso, foi omitida, no banco de dados, qualquer informação que identificasse seus participantes.

\section{Resultados}

Das 150 crianças que compunham o banco de dados, 30 preencheram os critérios de participação no estudo. Destas, seis $(20 \%)$ apresentaram histórico de regressão de habilidades de linguagem oral, constituindo, portanto, os participantes deste estudo. Todas as seis crianças (100\%) apresentaram perda definitiva de alguma habilidade linguística (escore 2). A média de idade dessas crianças, no momento da administração da ADI-R, era de 4,94 anos $(D P=0,46)$, sendo que todas, com exceção de uma, eram do sexo masculino.

\section{Características da Regressão Desenvolvimental}

Especificamente em relação à regressão das habilidades de linguagem oral, a média de idade de início da perda foi de 25 meses, variando de 12 meses a 32 meses, com uma duração média de 16,3 meses, sendo de no mínimo 6 meses e no máximo de 36 meses. Quanto ao nível de linguagem 
antes da perda, cinco crianças faziam uso comunicativo de pelo menos cinco palavras de forma espontânea e frequente; e uma utilizava palavras espontaneamente apenas de maneira ocasional. Ademais, duas crianças pararam de utilizar palavras para se comunicar com outras pessoas (intenção comunicativa).

Foi relatado que quatro crianças que tiveram regressão da linguagem oral também perderam habilidades de interação social, como por exemplo, interesse e envolvimento social. As outras duas crianças não apresentaram perda em outras áreas, além da linguagem. A média de idade de início da perda de habilidades sociais foi de 22,5 meses, variando de 18 meses a 30 meses, com duração média de 22 meses, sendo de no mínimo 18 meses e no máximo de 34 meses. Não se observou coocorrência entre a perda de habilidades de linguagem e doenças orgânicas, como a epilepsia em nenhuma das crianças.

\section{Interação Social}

$\mathrm{O}$ algoritmo da ADI-R conta com 16 itens referentes à dimensão de interação social. A Tabela 1 apresenta cada um desses itens e a distribuição das seis crianças nos escores correspondentes, em termos de frequência. De forma geral, observou-se que as seis crianças com regressão da linguagem oral apresentaram maior comprometimento no item que avalia a habilidade da criança em mostrar e direcionar a atenção do outro para compartilhar e naquele referente à brincadeira em grupo com outras crianças.

Assim, quanto ao direcionamento espontâneo da atenção do outro para compartilhar interesse, foi relatado que uma criança raramente ou nunca apresentava aproximações desse tipo (escore 3), quatro mostravam algum objeto para os pais, mas associado a preocupações ou necessidade de ajuda (escore 2); e apenas uma costumava mostrar coisas de seu interesse e direcionar a atenção do outro para compartilhar, porém com frequência limitada (escore 1). Em relação à brincadeira em grupo com outras crianças, foi mencionado que uma criança não procurava brincadeiras que envolvessem grupos, embora brincasse de correr ou de pegar (escore 3), quatro gostavam de atividades paralelas, mas com pouca ou nenhuma cooperação com as outras crianças (escore 2); e uma apresentava algumas brincadeiras cooperativas, mas com flexibilidade, frequência ou variedade limitada (escore 1).

\section{Linguagem e Comunicação}

$\mathrm{O}$ algoritmo da ADI-R conta com 13 itens referentes à dimensão de linguagem e comunicação. A Tabela 2 apresenta cada um desses itens e a distribuição das seis crianças nos escores correspondentes, em termos de frequência. De maneira geral, nessa dimensão, as seis crianças com regressão da linguagem oral apresentaram maior comprometimento nos itens que investigam o diálogo social, conversação recíproca, aceno com a cabeça para dizer 'sim' e imitação espontânea de ações. Os dois primeiros itens foram avaliados em cinco das seis crianças, visto que uma delas ainda não apresentava uso funcional da linguagem, enquanto que os outros dois foram investigados nas seis crianças.

Dessa forma, em relação ao diálogo social, foi mencionado que quatro crianças falavam para expressar necessidades, mas com pouco interesse social (escore 2); e apenas uma tinha algum uso social da fala em resposta ao interlocutor (escore 1). De forma semelhante, a conversação recíproca mostrouse pouco frequente (escore 2) em quatro crianças e somente uma apresentava esse comportamento ocasionalmente, mas com flexibilidade e tópicos limitados (escore 1).

Tabela 1. Distribuição dos Participantes de Acordo com os Escores dos Itens da Dimensão de Interação Social

\begin{tabular}{|c|c|c|c|c|}
\hline Item & $\begin{array}{c}\text { Escore } 0 \\
\text { n }\end{array}$ & $\begin{array}{c}\text { Escore } 1 \\
n\end{array}$ & $\begin{array}{c}\text { Escore } 2 \\
n\end{array}$ & $\begin{array}{c}\text { Escore } 3 \\
\mathbf{N}\end{array}$ \\
\hline Uso do corpo de outras pessoas para se comunicar & 1 & 4 & - & 1 \\
\hline Jogos imaginativos com outras crianças & - & 3 & 2 & 1 \\
\hline Contato visual direto & 2 & 3 & 1 & - \\
\hline Sorriso social & 1 & 5 & - & - \\
\hline Mostrando e direcionando atenção & - & 1 & 4 & 1 \\
\hline Oferecimento para compartilhar & - & 3 & 2 & 1 \\
\hline Busca de compartilhamento de prazeres com outros & 2 & 2 & 2 & - \\
\hline Oferecimento de conforto & 2 & 2 & 1 & 1 \\
\hline Qualidade da interação social & - & 6 & - & - \\
\hline Variação da expressão facial utilizada para comunicação & - & 1 & 2 & - \\
\hline Expressões faciais inadequadas & 1 & 3 & 2 & - \\
\hline Adequação das respostas sociais & - & 4 & 2 & - \\
\hline Interesse em crianças & 1 & 3 & 1 & 1 \\
\hline Resposta à aproximação de outras crianças & 1 & 3 & 2 & - \\
\hline Brincadeira em grupo com outras crianças & - & 1 & 4 & 1 \\
\hline Amizades & - & - & 1 & 1 \\
\hline
\end{tabular}


Os comportamentos descritos a seguir referem-se a aspectos da comunicação não-verbal e foram investigados nos seis casos com histórico de regressão da linguagem oral. Assim, o uso do gesto de assentir com a cabeça para dizer "sim" se mostrou ausente (escore 2 ) em cinco crianças e presente (escore 0) somente em uma. No que se refere à imitação espontânea de ações, foi informado que uma criança raramente imitava espontaneamente (escore 3), três apresentavam imitações espontâneas limitadas a algumas rotinas familiares (escore 2) e duas demonstravam alguma indicação de imitação espontânea de ações, mas com flexibilidade reduzida (escore 1).

\section{Comportamentos Repetitivos e Estereotipados}

$\mathrm{O}$ algoritmo da ADI-R conta com oito itens referentes à dimensão de comportamentos repetitivos e estereotipados. A Tabela 3 apresenta cada um desses itens e a distribuição das seis crianças nos escores correspondentes, em termos de frequência. De forma geral, as seis crianças apresentaram maior comprometimento nos itens que avaliam o uso repetitivo de objetos e maneirismos complexos do corpo.

Assim, quanto ao uso repetitivo de objetos ou ao interesse em partes destes, foi relatado que uma criança brincava utilizando os objetos de forma altamente repetitiva, interferindo de forma significativa em outras atividades (escore 3 ); três também usavam objetos de maneira fortemente repetitiva em brincadeiras, mas sem restringir suas atividades (escore 2); uma apresentava algum uso repetitivo de objetos, mas sem prejuízo social (escore 1); e uma tinha pouco uso repetitivo de objetos (escore 0 ). Já outros maneirismos complexos ou movimentos estereotipados do corpo foram informados como estando presentes de forma frequente, mas sem causar aflição se interrompidos (escore 2 ), em quatro crianças e ocasionais (escore 1) em duas.

Como pode ser observado, existe uma grande variabilidade na expressão da sintomatologia das crianças participantes do presente estudo. Contudo, elas compartilham alguns comprometimentos nas três dimensões que definem o diagnóstico do TEA.

Tabela 2. Distribuição dos Participantes de Acordo com os Escores dos Itens da Dimensão de Linguagem e Comunicação

\begin{tabular}{|c|c|c|c|c|}
\hline Item & $\begin{array}{c}\text { Escore } 0 \\
\mathbf{n}\end{array}$ & $\begin{array}{c}\text { Escore } 1 \\
n\end{array}$ & $\begin{array}{c}\text { Escore } 2 \\
n\end{array}$ & $\begin{array}{c}\text { Escore } 3 \\
\mathbf{N}\end{array}$ \\
\hline Elocuções estereotipadas e ecolalia tardia & - & 3 & 2 & - \\
\hline Diálogo social e conversa & - & 1 & 4 & - \\
\hline Conversação recíproca & - & 1 & 4 & - \\
\hline Questões ou colocações inapropriadas & 6 & - & - & - \\
\hline Inversão pronominal & 3 & - & 2 & - \\
\hline Neologismos / Linguagem idiossincrática & 3 & 2 & - & - \\
\hline Apontar para expressar interesse & 1 & 3 & 2 & - \\
\hline Aceno com a cabeça para dizer "sim" & 1 & - & 5 & - \\
\hline Aceno com a cabeça para dizer "não" & 2 & 2 & 2 & - \\
\hline Gestos convencionais / instrumentais & 2 & 3 & 1 & - \\
\hline Imitação espontânea de ações & - & 2 & 3 & 1 \\
\hline Brincar imaginativo & 1 & 4 & 1 & - \\
\hline Jogos de imitação social & - & 6 & - & - \\
\hline
\end{tabular}

Tabela 3. Distribuição dos Participantes de Acordo com os Escores dos Itens da Dimensão de Comportamentos Repetitivos e Estereotipados

\begin{tabular}{|c|c|c|c|c|}
\hline Item & $\begin{array}{c}\text { Escore } 0 \\
\text { n }\end{array}$ & $\begin{array}{c}\text { Escore } 1 \\
n\end{array}$ & $\begin{array}{c}\text { Escore } 2 \\
n\end{array}$ & $\begin{array}{c}\text { Escore } 3 \\
\mathbf{N}\end{array}$ \\
\hline Rituais verbais & 1 & 2 & 2 & - \\
\hline Preocupações não usuais & 6 & - & - & - \\
\hline Interesses restritos & 2 & 2 & 2 & - \\
\hline Uso repetitivo de objetos & 1 & 1 & 3 & 1 \\
\hline Compulsões / rituais & 5 & 1 & - & - \\
\hline Interesses sensoriais incomuns & 1 & 2 & 3 & - \\
\hline Maneirismos de dedos e mãos & 2 & 2 & 2 & - \\
\hline Outros maneirismos complexos ou movimentos estereotipados do corpo & - & 2 & 4 & - \\
\hline
\end{tabular}




\section{Discussão}

Os resultados demonstraram que $20 \%$ das crianças que compunham o banco de dados examinado apresentaram regressão das habilidades de linguagem oral nos primeiros três anos de vida. Esse achado se aproxima da percentagem encontrada em alguns estudos anteriores (Baird et al., 2008; Meilleur \& Fombonne, 2009; Siperstein \& Volkmar, 2004; Pickles et al., 2009). Entretanto, observa-se que ainda não há um consenso quanto à prevalência de regressão desenvolvimental no TEA, já que vários fatores parecem influenciar seu estabelecimento, como a definição operacional, o tamanho da amostra e o subgrupo estudado (Hansen et al., 2008; Rogers, 2004; Stefanatos, 2008).

Além disso, não se identificou coocorrência entre regressão da linguagem oral e epilepsia, em consonância com outras pesquisas (Baird et al., 2008; Christopher et al., 2004; Hansen et al., 2008; Jones \& Campbell, 2010), embora essa associação tenha sido demonstrada em alguns estudos (Valente \& Valério, 2004). Por outro lado, a coocorrência entre regressão da linguagem oral e perda de habilidades sociais foi observada na maioria das crianças, o que também foi constatado em estudos anteriores (Baird et al., 2008; Goldberg et al., 2003; Hansen et al., 2008; Luyster et al., 2005). A abordagem sociopragmática (Tomasello, 1999/2003) enfatiza a inter-relação entre as habilidades linguísticas e sociais, postulando que a aquisição da linguagem está atrelada a uma base interacionista, que começa a constituirse ainda no período pré-linguístico. Portanto, parece lógico pensar que a regressão da linguagem oral seria acompanhada de perda de habilidades sociais. Entretanto, os pais de duas das crianças participantes do presente estudo informaram apenas perda de linguagem. Acredita-se que isso se deve ao fato de a ADI-R investigar amplamente a regressão da linguagem, com base em nove itens específicos, ao passo que a perda de habilidades motoras, de interação social, de brincadeira e de autocuidado é acessada em conjunto a partir do mesmo número de itens. Portanto, a perda de habilidades sociais é menos explorada no instrumento, podendo este não ser sensível a sua ocorrência.

Outro aspecto relevante diz respeito à média de idade de início da regressão de linguagem oral nos casos estudados, que foi de 25 meses, e corroborou estudos anteriores (Baird et al., 2008; Meilleur \& Fombonne, 2009). Sendo assim, ao se considerar os passos desenvolvimentais da aquisição da linguagem propostos por Tomasello (1999/2003), quando ocorreu a regressão da linguagem oral essas crianças estariam adquirindo construções verbais insuladas, ou seja, expressões linguísticas organizadas em torno de um verbo. Estas, por sua vez, se fariam presentes em cenas experienciais nas quais há a marcação simbólica dos participantes e seus respectivos papéis, isto é, agente, paciente e instrumentos envolvidos na ação. Desta forma, teoricamente, no momento da regressão da linguagem oral os participantes do presente estudo já dominavam as habilidades de linguagem mais básicas e estavam adquirindo construções mais complexas, ou seja, desenvolvendo o que Tomasello (1999/2003) denomina de cognição linguística. De fato, antes da perda todas as crianças apresentavam linguagem funcional e espontânea, embora a utilizassem com frequências diferentes.
No que tange à caracterização dos comportamentos referentes à sintomatologia do TEA, observou-se que algumas habilidades mostraram-se mais comprometidas no grupo estudado. Nesse sentido, na dimensão de interação social, o direcionamento espontâneo da atenção de outras pessoas e a brincadeira em grupo com pares mostraram-se mais afetadas.

O item que avalia o direcionamento espontâneo da atenção tem como foco o compartilhamento de interesse, sendo que os resultados demonstraram que apenas uma criança participante do estudo costumava direcionar a atenção do adulto para compartilhar, mas com frequência limitada. Conforme Tomasello (1999/2003), compartilhar significa que ambos os agentes da interação prestam atenção tanto ao referente quanto ao estado de atenção do outro ao referente. Portanto, observa-se que a dimensão avaliada por esse item relaciona-se, diretamente, à habilidade de $\mathrm{AC}$, que concerne a situações de interação social triádica em que dois parceiros sociais dividem experiências quanto a um referente externo (e.g., objeto, evento), regulando seu estado de atenção (Tomasello, 1999/2003). Para o autor, as crianças passam a se envolver em cenas de AC quando começam a compreender o outro como um agente intencional, assim como elas próprias, sendo que o direcionamento da atenção do outro é o comportamento mais complexo da habilidade de $\mathrm{AC}$, pois envolve a iniciativa espontânea da criança, estabelecendose entre os 13 e os 15 meses. Nessa assertiva, alterações na referida habilidade são comuns em crianças com TEA e constituem-se em um importante preditor do transtorno (Bosa, 2002; Zanon, 2012), tendo sido investigadas por inúmeras pesquisas tanto nacional quanto internacionalmente (Farah, Perissinoto, \& Chiari, 2009; Hurwitz, 2010). Desta forma, há indícios de que os participantes do presente estudo apresentam comprometimento na habilidade de $\mathrm{AC}$, fundamental ao desenvolvimento sociocomunicativo.

De forma semelhante, a brincadeira em grupo com outras crianças também se mostrou comprometida no grupo estudado, sendo informado que apenas uma criança apresentava brincadeiras cooperativas, porém com flexibilidade e frequência limitadas. Empiricamente é bastante difundida a ideia de que crianças com TEA apresentam déficits na brincadeira, especialmente na sua função simbólica, o que se reflete diretamente na participação dessas crianças em brincadeiras cooperativas com pares (Fiaes \& Bichara, 2009; Hobson et al., 2009; Jahr, Eldevik, \& Eikeseth, 2000). Em termos sociopragmáticos, pode-se pensar que brincar com outras crianças envolve a compreensão dos parceiros sociais como agentes dotados de intencionalidade, o que possibilita perceber as estratégias comportamentais e as metas às quais se destinam (Tomasello, 1999/2003). Desta forma, tendo em vista que crianças com TEA têm déficits nessa compreensão, torna-se pertinente o achado de que as brincadeiras em grupo apresentam-se comprometidas. Além disso, para Tomasello (1999/2003) o ato de brincar com pares exige flexibilidade, já que a criança precisa considerar que há um contexto representacional comum no qual estão implícitas diferentes perspectivas que abrangem objetivos e intenções diversas, sendo que essa flexibilidade é influenciada, diretamente, pela capacidade da criança de operar com símbolos linguísticos. Portanto, a suposta dificuldade que os participantes do 
presente estudo tem quanto à compreensão dos outros como agentes intencionais gera déficits importantes nas suas aptidões simbólicas. Esses déficits, por sua vez, causam limitações na flexibilidade cognitiva, exigida em atividades como a brincadeira em grupo.

No que se refere à dimensão de comunicação, as habilidades mais comprometidas nas crianças participantes desse estudo dizem respeito ao diálogo social, à conversação recíproca, ao aceno com a cabeça para dizer 'sim' e à imitação espontânea de ações. Tendo em vista a natureza pragmática comum do diálogo social e da conversação, ambos serão abordados em conjunto, porém é importante expor que as diferenças na definição operacional desses itens na ADI-R concernem à aproximação social (diálogo) e à reciprocidade social (conversação). Observa-se, então, que o primeiro aspecto encontra-se implicado no segundo, já que não há reciprocidade sem aproximação, sendo que os resultados demonstraram que ambos os comportamentos apresentaram-se presentes em apenas uma criança, porém de forma ocasional e com flexibilidade limitada.

Tanto o diálogo social quanto a conversação recíproca envolvem os aspectos pragmáticos da linguagem, isto é, o uso desta em sua função sociocomunicativa. Nesse sentido, o comprometimento da habilidade pragmática é considerado um traço comum em indivíduos com TEA(Eigsti, Marchena, Schuh, \& Kelley, 2011), que podem apresentar alterações nas funções comunicativas (e.g., protesto, pedido), nas habilidades conversacionais (e.g., obedecer às trocas de turno) e nos meios comunicativos: gestual, vocal e verbal (Brito \& Carrara, 2010; Fernandes, 1996). Levando em consideração os pressupostos da teoria de Tomasello (1999/2003), pode-se afirmar que fazer uso de suas habilidades pragmáticas implica flexibilidade cognitiva, já que as crianças envolvidas no intercâmbio comunicativo devem adaptar os enunciados aos conhecimentos, expectativas e focos de atenção do parceiro, compreendendo, portanto, os papéis desempenhados pelo falante e pelo ouvinte. Faz-se necessário, então, que a criança determine a intenção comunicativa do interlocutor, atribuindo diferentes papéis aos elementos linguísticos empregados à medida que os turnos conversacionais se alternam (Tomasello, 1999/2003). Desta forma, observa-se que a habilidade pragmática está atrelada à capacidade da criança de perceber a si e ao outro como agentes intencionais, sendo esta uma dificuldade característica de indivíduos com TEA, conforme referido anteriormente.

Além das dificuldades pragmáticas, observou-se comprometimento na habilidade de acenar com a cabeça para dizer 'sim', sendo mencionado que apenas uma criança participante da presente pesquisa utilizava esse gesto. Embora as alterações na linguagem oral sejam um traço comum de crianças com TEA, frequentemente se observam, também, comprometimentos na comunicação gestual (Prestes, Tamanaha, \& Perissinoto, 2009; Sowden, Perkins, \& Clegg, 2008). Com base na perspectiva sociopragmática, a aquisição do gesto se dá por imitação, sendo necessário que a criança compreenda a intenção do adulto ao usar determinado ato gestual para que ela possa, então, utilizá-lo na mesma função comunicativa, criando um elemento gestual intersubjetivo (Tomasello, 1999/2003).
Desse modo, observa-se que o uso de gestos e a habilidade imitativa encontram-se atrelados, o que se reflete no fato de a imitação espontânea de ações ter se demonstrado igualmente comprometida nos participantes desse estudo. Foi informado que apenas duas crianças apresentavam alguma indicação de imitação espontânea, mas com flexibilidade reduzida. De forma semelhante aos déficits pragmáticos, os comprometimentos na habilidade imitativa parecem ser comuns em indivíduos com TEA (ver Timo, Maia, \& Ribeiro, 2011, para uma revisão). Do ponto de vista sociopragmático, a aprendizagem por imitação é uma das principais formas de aprendizagem cultural humana, na qual as crianças tentam se colocar no espaço intencional do outro para, então, discernir seu objetivo ao utilizar determinado artefato (Tomasello, 1999/2003). Ressalta-se que, nessa perspectiva, a imitação refere-se ao comportamento intencional do parceiro social e não apenas à reprodução dos efeitos de suas ações. Para o autor, a capacidade imitativa "abre as portas" para formas cognitivamente mais significativas de aprendizagem. Portanto, é possível ponderar que os déficits na imitação de crianças com TEA alteram suas vias de aprendizagem cultural, comprometendo seu desenvolvimento linguístico e cognitivo.

Afora as dimensões de interação social e comunicação, na área de comportamentos restritos e repetitivos também se observou uma tendência do grupo estudado, no que se refere aos déficits mais significativos. Essa área difere das outras duas dimensões, pois aborda, especificamente, sintomas do TEA. Sendo assim, os itens da ADI-R que medem o uso repetitivo de objetos e maneirismos complexos ou movimentos estereotipados do corpo mostraram-se mais comprometidos nos participantes da presente pesquisa. Conforme referido, ambos os comportamentos compõem a sintomatologia do TEA, embora não se apresentem uniformemente, podendo inclusive modificar-se com a idade (Esbensen, Seltzer, Lam, \& Bodfish, 2009). Movimentos complexos do corpo são comportamentos estereotipados e voluntários que envolvem diferentes partes do corpo (e.g., levantar e abaixar os braços enquanto caminha na ponta dos pés), sendo que, embora possam estar presentes em outras condições, o padrão elevado de ocorrência e a gravidade com que se apresentam parecem ser característicos do TEA (Bodfish, Symons, Parker, \& Lewis, 2000; Lord et al., 1994).

No que tange ao uso repetitivo de objetos, diversos estudos têm demonstrado que esse comportamento é frequente em crianças com TEA, podendo se apresentar precocemente (Ozonoff et al., 2008). Desta forma, tendo como base a teoria sociopragmática, sabe-se que após os 9 meses de idade as crianças passam a ver alguns objetos além de suas características sensoriomotoras, aprendendo a interagir intencionalmente com eles. Tal fato se dá porque, ao compreenderem o outro como agente intencional, elas aprendem, por imitação, o uso convencional de objetos, sendo que entre os 18 e os 24 meses as crianças começam a utilizar objetos como símbolos, demonstrando indícios de brincadeira simbólica (Tomasello, 1999/2003). Portanto, depreende-se que a dificuldade que os participantes dessa pesquisa têm na compreensão do outro como ser intencional, gera déficits na capacidade imitativa e perspectiva, que, por fim, parece se refletir no uso repetitivo de objetos. 
Assim, a repetição demonstra que o indivíduo concebe os objetos a partir de um único prisma, e não de múltiplas perspectivas. Entretanto, dada a complexidade dos comportamentos repetitivos e estereotipados, é necessário levar em consideração outros tipos de explicações como, por exemplo, alterações neuroanatômicas (Pierce \& Courchesne, 2001) e neurobiológicas (Leekman, Prior, \& Uljarevic, 2011).

\section{Considerações Finais}

No que diz respeito à regressão da linguagem, observa-se que, apesar do número reduzido de participantes, os achados do presente estudo corroboraram pesquisas anteriores no que tange à prevalência de regressão, à idade de início da perda, à associação com perda de habilidades de interação social, entre outros fatores. Tais achados reforçam que, ao contrário do que se postulava em décadas anteriores, a regressão não é fruto da imaginação parental, mas sim um fenômeno fidedigno que afeta uma parcela importante de crianças posteriormente diagnosticadas com TEA. Entretanto, a regressão de habilidades de linguagem permanece pouco compreendida, não apenas quanto a sua etiologia, mas também do ponto de vista teórico, o que reforça a importância investigativa do tema em futuros estudos.

Ademais, a análise da sintomatologia à luz da teoria sociopragmática permitiu perceber características em comum entre os comprometimentos mais significativos da tríade sintomatológica do TEA (interação social, comunicação e comportamentos repetitivos e estereotipados). Nessa assertiva, destaca-se a dificuldade que as crianças com o transtorno têm em compreender os outros como agentes intencionais, o que parece estar no cerne de seus déficits na habilidade de AC, na imitação, na brincadeira com pares e na pragmática. Desta forma, a abordagem sociopragmática fornece um modelo explanatório plausível acerca da interface entre diferentes áreas do desenvolvimento infantil e suas alterações.

No entanto, cabe ressaltar que a presente pesquisa apresenta limitações que necessitam ser explicitadas. Uma delas diz respeito ao tamanho reduzido do grupo estudado, decorrente da perda amostral ocasionada pela quantidade significativa de participantes com dados incompletos nas medidas investigadas. Outra limitação refere-se ao uso da ADI-R. Este, por ser um instrumento administrado aos pais ou cuidadores, depende, fundamentalmente, da capacidade destes em recordar eventos passados que permitam a codificação da informação na ADI-R. Portanto, não há observação direta do comportamento da criança. Relacionado a esse aspecto, encontra-se o fato do uso do banco de dados restringir o acesso a informações complementares que poderiam favorecer o entendimento acerca de determinados comportamentos no grupo estudado, como por exemplo, a ocorrência de eventos traumáticos na família antes da regressão (morte/doença de pessoas próximas à criança), a escolaridade dos pais e o nível cognitivo da criança.

De qualquer forma, destaca-se a importância de se investigar a sintomatologia do TEA com base em instrumentos reconhecidos internacionalmente, que ainda não estão disponíveis para uso no Brasil, como é o caso da ADI-R, justificando o uso desse banco de dados. Sugere-se, também, que pesquisas futuras sejam realizadas com amostras maiores e incluindo grupo controle (no caso crianças com TEA e sem histórico de regressão de linguagem), com o intuito de estender os achados preliminares do presente estudo.

\section{Referências}

American Psychiatric Association. (2013). Diagnostic and Statistical Manual of Mental Disorders (5th ed.). Arlington, VA: American Psychiatric Publishing.

Backes, B. (2012). Regressão da linguagem, desenvolvimento sociocomunicativo e perfil sintomatológico de crianças com transtorno do espectro autístico (Unpublished master's thesis). Universidade Federal do Rio Grande do Sul, Porto Alegre, Rio Grande do Sul, Brasil.

Baird, G., Charman, T., Pickles, A., Chandler, S., Loucas, T., Meldrum, D. Carcani-Rathwell, I., Serkana, D., \& Simonoff, E. (2008). Regression, developmental trajectory and associated problems in disorders in the autism spectrum: The SNAP study. Journal of Autism and Developmental Disorders, 38, 1827-1836.

Barger, B., Campbell, J. M., \& McDonough, J. (2013). Prevalence and onset of regression within autism spectrum disorders: A meta-analytic review. Journal of Autism and Developmental Disorders, 43(4), 817-828.

Bodfish, J. W., Symons, F. J., Parker, D. E., \& Lewis, M. H. (2000). Varieties on repetitive behavior in autism: Comparisons to mental retardation. Journal of Autism and Developmental Disorders, 30(3), 237-243.

Bosa, C. (2002). Atenção compartilhada e identificação precoce do autismo. Psicologia: Reflexão e Crítica, 15(1), 77-88.

Brito, M. C., \& Carrara, K. (2010). Alunos com distúrbios do espectro autístico em interação com professores na educação inclusiva: Descrição de habilidades pragmáticas. Revista da Sociedade Brasileira de Fonoaudiologia, 15(3), 421-429.

Centers for Disease Controland Prevention (2014). Prevalence of autism spectrum disorder among children aged 8 years: Autism and developmental disabilities monitoring network. MMWR, 63(SS02), 1-21

Esbensen, A. J., Seltzer, M. M., Lam, K. S. L., \& Bodfish, J. W. (2009). Age-related differences in restricted repetitive behaviors in Autism Spectrum Disorders. Journal of Autism and Developmental Disorders, 39(1), 57-66.

Eigsti, I. M., Marchena, A. B., Schuh, J. M., \& Kelley, E. (2011). Language acquisition in autism spectrum disorders: A developmental review. Research in Autism Spectrum Disorders, 5, 681-691.

Farah, L. S. D., Perissinoto, J., \& Chiari, B. M. (2009). Estudo longitudinal da atenção compartilhada em crianças autistas não-verbais. Revista CEFAC, 11(14), 587-597.

Fernandes, F. D. M. (1996). Autismo infantil: Repensando o enfoque fonoaudiológico - Aspectos funcionais da comunicação. São Paulo: Lovise. 
Fiaes, C. S., \& Bichara, I. D. (2009). Brincadeira de faz-de-conta em crianças autistas: Limites e possibilidades numa perspectiva evolucionista. Estudos de Psicologia, 14(3), 231-238.

Fombonne, E. (2009). Epidemiology of pervasive developmental disorders. Pediatric Research, 65(6), 591-598.

Goldberg, W. A., Osann, K.,Filipek, P. A., Laulhere, T., Jarvis, K., Modahl, C., Flodman, P., \& Spence, M. A. (2003). Language and other regression: Assessment and timing. Journal of Autism and Developmental Disorders, 33(6), 607-616.

Hansen, R. L., Ozonoff, S., Krakowiak, P., Angkustsiri, K., Jones, C., Deprey, L. J., Le, D. N., Croen, L. A., \& Hertz-Picciotto, I. (2008). Regression in autism: Prevalence and associated factors in the CHARGE study. Ambulatory Pediatrics, 8(1), 25-31.

Hobson, R. P., Lee, A., \& Hobson, J. A. (2009). Qualities of symbolic play among children with autism: A social-developmental perspective. Journal of Autism and Developmental Disorders, $39,12-22$.

Hurwitz, S. (2010). Joint attention revisited: Examining heterogeneity among children with autism (Unpublished doctoral dissertation). Doctorate in Philosophy, School of Education, University of North Carolina at Chapel Hill., North Carolina, United States of America.

Jahr, E., Eldevik, S., \& Eikeseth, S. (2000). Teaching children with autism to initiate and sustain cooperative play. Research on Development Disabilities, 21(2), 151-169.

Jones, L. A., \& Campbell, J. M. (2010). Clinical characteristics associated with language regression for children with autism spectrum disorders. Journal of Autism and Developmental Disorders, 40, 54-62.

Klinger, L., Dawson, G., \& Renner, P. (2002). Autistic disorder. In E. Mash \& R. Barkley (Eds.), Child psychopathology (2nd ed., pp. 409-454). New York: Guilford Press.

Landa, R. (2007). Early communication development and intervention for children with autism. Mental Retardation and Developmental Disabilities Research Reviews, 13(1), 16-25.

Leekman, S. R., Prior, M. R., \& Uljarevic, M. (2011). Restricted and repetitive behaviors in autism spectrum disorders: A review of research in the last decade. Psychological Bulletin, 137(4), 562-593.

Lord, C., Rutter, M., \& Le Couteur, A. (1994). Autism Diagnostic Interview-Revised: A revised version of a diagnostic interview for caregivers of individuals with possible pervasive developmental disorders. Journal of Autism and Developmental Disorders, 24(5), 659-685.

Lord, C., Shulman, C., \& DiLavore, P. (2004). Regression and word loss in autistic spectrum disorders. Journal of Child Psychology and Psychiatry, 45(5), 936-955.

Luyster, R., Richler, J.,Risi, S., Hsu, W., Dawson, G., Bernier, R., ... Lord, C (2005). Early regression in social communication in autism spectrum disorders: a CPEA study. Developmental Neuropsychology, 27(3), 311-336.

Matson, J. L., \& Kozlowski, A. M. (2010). Autistic regression. Research in Autism Spectrum Disorders, 4, 340-345.

McConkey, R., Truesdale-Kennedy, M., \& Cassidy, A. (2009). Mothers' recollections of early features of autism spectrum disorders. Child and Adolescent Mental Health, 14(1), 31-36.
Meilleur, A. A. S., \& Fombonne, E. (2009). Regression of language and non-language skills in pervasive developmental disorders. Journal of Intellectual Disability Research, 53(2), 115-124.

Molloy, C. A., Keddache, M., \& Martin, L. J. (2005). Evidence for linkage on $21 \mathrm{q}$ and $7 \mathrm{q}$ in a subset of autism characterized by developmental regression. Molecular Psychiatry, 10, 741-746.

Ozonoff, S., Macari, S., Young, G. S., Goldring, S., Thompson, M., \& Rogers, S. J. (2008). Atypical object exploration at 12 months of age is associated with autism in a prospective sample. Autism, 12, 457-472.

Pickles, A., Simonoff, E., Conti-Ramsden, G., Falcaro, M., Simkin, Z., Charman, T., Chandler, S., Loucas, T., \& Baird, G. (2009). Loss of language in early development of autism and specific language impairment. Journal of Child Psychology and Psychiatry, 50(7), 843-852.

Pierce, K., \& Courchesne, E. (2001). Evidence for a cerebellar role in reduced exploration and stereotyped behavior in autism. Biological Psychiatry, 49(8), 655-664.

Prestes, R., Tamanaha, A. C., \& Perissinoto, J. (2009). Uso do gesto no transtorno autista: Estudo de caso único. Revista CEFAC, 11(4), 708-712.

Rapin, I., \& Dunn, M. (2003). Update on the language disorders of individuals on the autistic spectrum. Brain and Development, $25,166-172$.

Rogers, S. J. (2004). Developmental regression in autism spectrum disorders. Mental Retardation \& Developmental Disabilities Research Reviews, 10, 139-143.

Rutter, M. L. (2011). Progress in understanding autism: 20072010. Journal of Autism and Developmental Disorders, 41(4), 395-404.

Siperstein, R., \& Volkmar, F. (2004). Brief report: parental reporting of regression in children with pervasive developmental disorders. Journal of Autism and Developmental Disorders, 34(6), 731-734

Sowden, H., Perkins, M., \& Clegg, J. (2008). The co-development of speech and gesture in children with autism. Clinical Linguistics \& Phonetics, 22(10-11), 804-813.

Stefanatos, G. A. (2008). Regression in autistic spectrum disorders. Neuropsychology Review, 18, 305-319.

Timo, A. L. R., Maia, N. V. R., \& Ribeiro, P. C. (2011). Déficit de imitação e autismo: Uma revisão. Psicologia USP, 22(4), 833-850.

Tomasello, M. (2003). Origens culturais da aquisição do conhecimento humano. (C. Berliner, Trans.) São Paulo: Martins Fontes. (Original published in 1999)

Valente, K. D. R., \& Valério, R. M. (2004). Transtorno invasivo do desenvolvimento e epilepsia. Journal of Epilepsy and Clinical Neurophysiology, 10(4), 41-46.

Tuchman, R. F. (2006). Autism and epilepsy: What has regression got to do with it? Epilepsy Currents, 6(4), 107-111.

Volden, J., Coolican, J., Garon, N., White, J., \& Bryson, S. (2009). Brief Report: Pragmatic language in Autism Spectrum Disorder: Relationships to measures of ability and disability. Journal of Autism and Developmental Disorders, 39, 388-393.

Wiggins, L. D., Rice, C. E., \& Baio, J. (2009). Developmental regression in children with an autism spectrum disorder identified by a population-based surveillance system. Autism, 13(4), 357-374. 
Zanon, R. B. (2012). Déficit na iniciativa e na resposta de atenção compartilhada como principal preditor de comportamento social no transtorno do espectro autista (Unpublished master's thesis). Universidade Federal do Rio Grande do Sul, Porto Alegre, Rio Grande do Sul.
Zhang, Y., Xu, Q., Liu J., Li S., \& Xu, X. (2012). Risk factors for autistic regression: Results of an ambispective cohort study. Journal of Child Neurology, 27(1). Retrieved from http://jen. sagepub.com/content/early/2012/01/21/0883073811430163

Recebido em 18.12.2013

Primeira decisão editorial em 03.08.2015

Aceito em 03.08.2015 